\title{
THE BREAKUP OF A LIQUID JET AT MICROGRAVITY
}

\author{
S.P. Lin and A. Honohan \\ Department of Mechanical and Aeronautical Engineering \\ Clarkson University, Potsdam, NY 13699
}

\begin{abstract}
The parameter ranges in which a viscous liquid jet emanating into a viscous gas will breakup in three different modes are calculated. The three modes of jet breakup are: Rayleigh mode of capillary pinching, Taylor mode of atomization, and the absolute instability mode. The aparatus designed for elucidating these three different modes of instability is described. Some preliminary results of the ongoing ground based experiments will be reported.
\end{abstract}

\section{INTRODUCTION}

We investigate the stability of core-annular flow with respect to spatially growing disturbances. Spatially growing disturbances are especially relevant to the atomization process which utilizes the amplification of disturbances as they are convected downstream to generate small droplets. Atomization is a process used in various industrial, applications which include fuel sprays, fire suppressing, and advanced material processing. The atomization process must be carried out outside of the Rayleigh regime [1] of jet breakup by capillary pinching. It must also avoid absolute instability [2,3]. The liquid core-gas annular flow of two viscous fluids is shown to be capable of exhibiting either absolute or convective instability depending on the specific values of flow parameters involved. The relevant parameters are the Weber number, the Reynolds number, the Froude number, the density ratio, the viscosity ratio, and the radius ratio. The type of instability depends most sensitively on We and Re. The critical Weber number below which the flow is convectively unstable and above which the flow is absolutely unstable, is obtained as a function of the Reynolds number of the given remaining parameters. There are two modes of convective instability. The Rayleigh mode of convective instability caused by capillary pinching and the Taylor mode of atomization caused by interfacial stress fluctuation. The Taylor mode instability is shown to change over to the Rayleigh mode when the Froude number is increased beyond a certain value with the rest of flow parameters fixed. The practical implication of this is that the atomization processes widely used on earth in a given parameter range may fail to produce droplets smaller than the jet thickness at microgravity. This is due to the reduction in the interfacial stress fluctuation in the liquid core-gas annular flow at microgravity. Thus we are given an opportunity to explore the fundamental dynamics of interface at microgravity. The experiment described herein is aiming at elucidating these theoretical concepts. Moreover, the experiment will enable us to ascertain the notion of absolute instability which is predicted in various theories neglecting the gravity.

\section{STABILITY ANALYSIS}

Consider the stability of a cylindrical liquid jet of density $\rho_{1}$ flowing vertically downward in the direction of gravitational acceleration $g$. The jet is surrounded by a gas of density $\rho_{2}$ occupying the annular space between the jet and the inner wall of a vertical pipe of radius $R_{2}$. 
The liquid and gas flows have the same pressure gradient in the jet flow direction. Hence the pressure drop across the liquid-gas interface at any cross section is constant along the axial direction. This pressure difference is balanced exactly by the surface tension force per unit surface area of the liquid cylinder of constant radius $R_{1}$ which is also the radius of curvature. This basic flow which satisfies exactly the Navier-Stokes equations can be written as [4]

$$
\begin{gathered}
W_{1}(r)=-1+\frac{N r^{2}}{\left[N-\left(1-\ell^{2}\right)\right]}\left\{1-\frac{1-Q}{4 N} \frac{R e}{F r}\left[2 \ln \ell+\left(1-\ell^{2}\right)\right]\right\}, \\
W_{2}(r)=\frac{\left(\ell^{2}-r^{2}\right)}{\left[N-\left(1-\ell^{2}\right)\right]}\left\{1-\frac{(1-Q)}{4 N} \frac{R e}{F r}\left[2 \ln \ell+\left(1-\ell^{2}\right)\right]\right\} \\
+\frac{(1-Q)}{4 N} \frac{R e}{F r}\left[\ell^{2}-r^{2}-2 \ln \left(\frac{\ell}{r}\right)\right] \\
N=\frac{\mu_{2}}{\mu_{1}}, \quad \ell=\frac{R_{2}}{R_{1}}, \quad Q=\frac{\rho_{2}}{\rho_{1}}
\end{gathered}
$$

where the subscript 1 or 2 stands for the liquid or the gas phase respectively, $r$ is the radial distance normalized with $R_{1}, W(r)$ is the axial velocity distribution, $\mu$ is the dynamic viscosity, $\rho$ is the density, and $W_{0}$ is the maximum jet velocity, $R e \equiv$ Reynolds number $=\rho_{1} W_{0} R_{1} / \mu_{1}$, and $\mathrm{Fr} \equiv$ Froude number $=\mathrm{W}_{\mathrm{o}}{ }^{2} / \mathrm{gr}_{1}$,

The axisymmetric normal mode disturbance is governed by the well known OrrSommerfeld equation in each phase. The solutions of the governing equations in the liquid and the gas phases are represented respectively by truncated series of two complete sets of orthogonal functions. These sets of functions satisfy the boundary conditions of zero radial velocity along the jet axis and on the inner wall of the vertical pipe. The other boundary conditions to be satisfied include the vanishing of tangential components of velocity at the pipe wall and the five conditions at the liquid-gas interface. The interfacial conditions are: balance of normal and tangential components of interfacial forces, continuity of normal and tangential velocity components, and a kinematic condition which relates the total time rate of displacement of the interface to the radial component of velocity. In addition to the five parameters introduced in the description of the basic state, there is an additional flow parameter appearing in this system. This additional parameter is the Weber number.

$$
W e=S / \rho_{1} W_{o}^{2} R_{1},
$$

which arises from the normal force balance at the interface. The Weber number represents the 
ratio of the surface tension force to the inertia force. For a given set of flow parameters, the set of homoegneous equations possess a non-trivial solution only for certain characteristic frequencies $\omega$ and wave numbers $\mathrm{k}$ of the normal mode disturbance. $\omega$ and $\mathrm{k}$ must satisfy the condition that the determinant of the coefficient matrix of the above system vanishes. This yields the characteristic equation given by equation (21) of Lin and Ibrahim [4]. The normal mode disturbances involve an exponential factor $\exp (\mathrm{ikz}+\omega \mathrm{t})$, where $\mathrm{t}$ is the time and $\mathrm{z}$ is the axial distance measured in the opposite direction of the liquid flow. Both $\mathrm{k}$ and $\omega$ are complex, i.e. $\mathrm{k}=\mathrm{k}_{\mathrm{r}}+\mathrm{ik}$, and $\omega=\omega_{\mathrm{r}}+\mathrm{i} \omega_{\mathrm{i}}$. Hence $\mathrm{k}_{\mathrm{i}}>0$ gives the spatial growth rate of disturbances convected as a group in the downstream direction. $\omega_{r}>0$ gives the temporal growth rate of unstable disturbances. $k_{r}$ is the real wave number which is inversely proportional to the wave length. $\omega_{i}$ is the natural frequency of oscillation of the normal mode disturbance, The group velocity of the disturbance is given by $-\partial \omega_{\mathrm{r}} / \partial \mathrm{k}_{\mathrm{r}}$. In the temporal formulation $\mathrm{k}$ is treated as real.

To determine the spatial amplification rate of convectively unstable disturbances for a given set of parameters, we put $\omega_{\mathrm{r}}=0$ and assign different values to $\omega_{\mathrm{i}}$ in the characteristic equations. $k_{r}$ and $k_{i}$ are then solved from the real and imaginary parts of the characteristic equation. The subrountines CGEDI and DNSQ in the SLATEC library are utilized to effect the solution. The set of points $\left(\mathrm{k}_{\mathrm{r}}, \mathrm{k}_{\mathrm{i}}\right)$ for various values of $\omega_{\mathrm{i}}$ determine the spatial amplification curve $\omega_{\mathrm{r}}=0$. The numerical results are reported elsewhere. The main findings are already summarized in the introduction and will not be repeated here.

\section{EXPERIMENTS}

The liquid jet is produced using the pressure vessel shown in Figure 1. Prior to the experiment the vessel is filled with liquid and compressed gas. The pressure inside the vessel is monitored with a pressure gauge at the top of the cylinder. To start the flow of liquid, a remotely operated solenoid valve is opened. The pressure difference between the inside of the vessel and the surrounding air forces the liquid through a nozzle connected to the solenoid valve. Different jet velocities can be obtained by varying the internal pressure of the vessel. This design was chosen over several alternatives because of its versatility. This single vessel will be capable of producing jets which exhibit all three modes of jet breakup, namely Rayleigh mode, Taylor mode and absolutely unstable mode. Table 1 delineates some estimated operating parameters for each mode. An estimation of the expected intact length of the jet is also presented in Table 1 .

Currently the design is limited by the solenoid valve to a maximum pressure of $60 \mathrm{psig}$. The same pressure vessel, with a different solenoid valve can be used at pressures up to about 150 psig. This operating pressure is sufficient to span much of the parameter range for Rayleigh mode and absolutely unstable mode, with Taylor mode requiring higher pressures.

Other components of the apparatus include a piezoelectric device, camera, and light source. A piezoelectric device, with the proper electronics, may be used in order to introduce disturbances of various frequencies into the liquid jet. The camera and light source will be used to record the stability characteristics. All of these components, including the pressure vessel, are contained within a single steel frame with dimensions of $50 \mathrm{~cm} \times 25 \mathrm{~cm} \mathrm{x} 76 \mathrm{~cm}$. 
One g experiments are currently being carried out at Clarkson University. Preliminary results for the one $g$ tests will be presented. The apparatus was designed so that it could be used for drop tower testing with a minimum amount of modification.

\section{ACKNOWLEDGEMENTS}

The work was supported in part by Grants No. NAG3-1402 of NASA, DAAL 03-89-K0179 of ARO.

\section{REFERENCES}

${ }^{1}$ S.P. Lin and Z.W. Lian, AIAA Journal 28, (1990), 120.

${ }^{2}$ S.J. Leib and M.E. Goldstein, Phys. Fluids 29, (1986), 952.

${ }^{3}$ S.P. Lin adn Z.W. Lian, Phys. Fluids A 1, (1989), 490.

${ }^{4}$ S.P. Lin and E.A. Ibrahim, J. Fluid Mech. 218, (1991), 641. 


\section{Table 1: Parameter Ranges for Liquid Jet Experiments}

for a nozzle diameter of $0.5 \mathrm{~mm}$

Absolute Instability

\begin{tabular}{||l||c|c|c|c|c|c||}
\hline Fluid & $U(\mathrm{~m} / \mathrm{s})$ & $\begin{array}{l}\text { Reynolds } \\
\text { Number (R) }\end{array}$ & $\begin{array}{l}\text { Weber } \\
\text { Number (We) }\end{array}$ & $\begin{array}{l}\text { Density } \\
\text { Ratio (Q) }\end{array}$ & $\begin{array}{l}\text { Intact } \\
\text { Length (cm) }\end{array}$ & $\begin{array}{l}\text { Pressure } \\
\text { (psig) }\end{array}$ \\
\hline SAE 10 Oil & 1.08 & 2.27 & 0.135 & 0.00134 & 1.5 & 26 \\
\hline SAE 10 Oil & 0.54 & 1.14 & 0.538 & 0.00134 & 0.7 & 13 \\
\hline SAE 30 Oil & 1.07 & 0.85 & 0.133 & 0.00134 & 3.0 & 69 \\
\hline SAE 30 Oil & 0.53 & 0.42 & 0.544 & 0.00134 & 1.5 & 34 \\
\hline SAE 30 Oil & 0.15 & 0.12 & 6.79 & 0.00134 & - & 9.5 \\
\hline Water & 0.75 & 190 & 0.500 & 0.0013 & 0.2 & 0.2 \\
\hline Water & 0.47 & 118 & 1.300 & 0.0013 & 0.1 & 0.1 \\
\hline Water & 0.15 & 37 & 13.00 & 0.0013 & - & 0.04 \\
\hline Ethanol & 0.85 & 140 & 0.16 & 0.0016 & - & 0.02 \\
\hline
\end{tabular}

Rayleigh Mode Instability

\begin{tabular}{|l|c|c|c|c|c|c||}
\hline Fluid & $\begin{array}{l}\mathrm{U} \\
(\mathrm{m} / \mathrm{s})\end{array}$ & $\begin{array}{l}\text { Reynolds } \\
\text { Number }(\mathrm{R})\end{array}$ & $\begin{array}{l}\text { Weber } \\
\text { Number (We) }\end{array}$ & $\begin{array}{l}\text { Density } \\
\text { Ratio (Q) }\end{array}$ & $\begin{array}{l}\text { Intact } \\
\text { Length (cm) }\end{array}$ & $\begin{array}{l}\text { Pressure } \\
(\mathrm{psig})\end{array}$ \\
\hline SAE 10 Oil & 10.83 & 22.8 & 0.00134 & 0.00134 & 25 & 272 \\
\hline SAE 10 Oil & 3.423 & 7.20 & 0.0134 & 0.00134 & 10 & 84 \\
\hline SAE 30 Oil & 10.67 & 8.44 & 0.00134 & 0.00134 & 2.0 & 680 \\
\hline SAE 30 Oil & 3.375 & 2.67 & 0.0134 & 0.00134 & 5.0 & 220 \\
\hline Water & 14.88 & 3720 & 0.0013 & 0.0013 & 3.8 & 23 \\
\hline Water & 4.71 & 1170 & 0.013 & 0.0013 & 1.3 & 3.1 \\
\hline Ethanol & 8.50 & 1398 & 0.0016 & 0.0016 & 2.5 & 7.5 \\
\hline
\end{tabular}

Taylor Mode Instability

\begin{tabular}{||l|l|l|l|l|l||}
\hline Fluid & $\mathrm{U}(\mathrm{m} / \mathrm{s})$ & $\begin{array}{l}\text { Reynolds } \\
\text { Number (R) }\end{array}$ & $\begin{array}{l}\text { Weber Number } \\
(\mathrm{We})\end{array}$ & $\begin{array}{l}\text { Density Ratio } \\
(\mathrm{Q})\end{array}$ & Pressure (psig) \\
\hline Water & 148.8 & 74400 & 0.00013 & 0.0013 & 3000 \\
\hline Water & 105.2 & 52600 & 0.00026 & 0.0013 & 1300 \\
\hline Water & 74.4 & 37200 & 0.00052 & 0.0013 & 680 \\
\hline
\end{tabular}



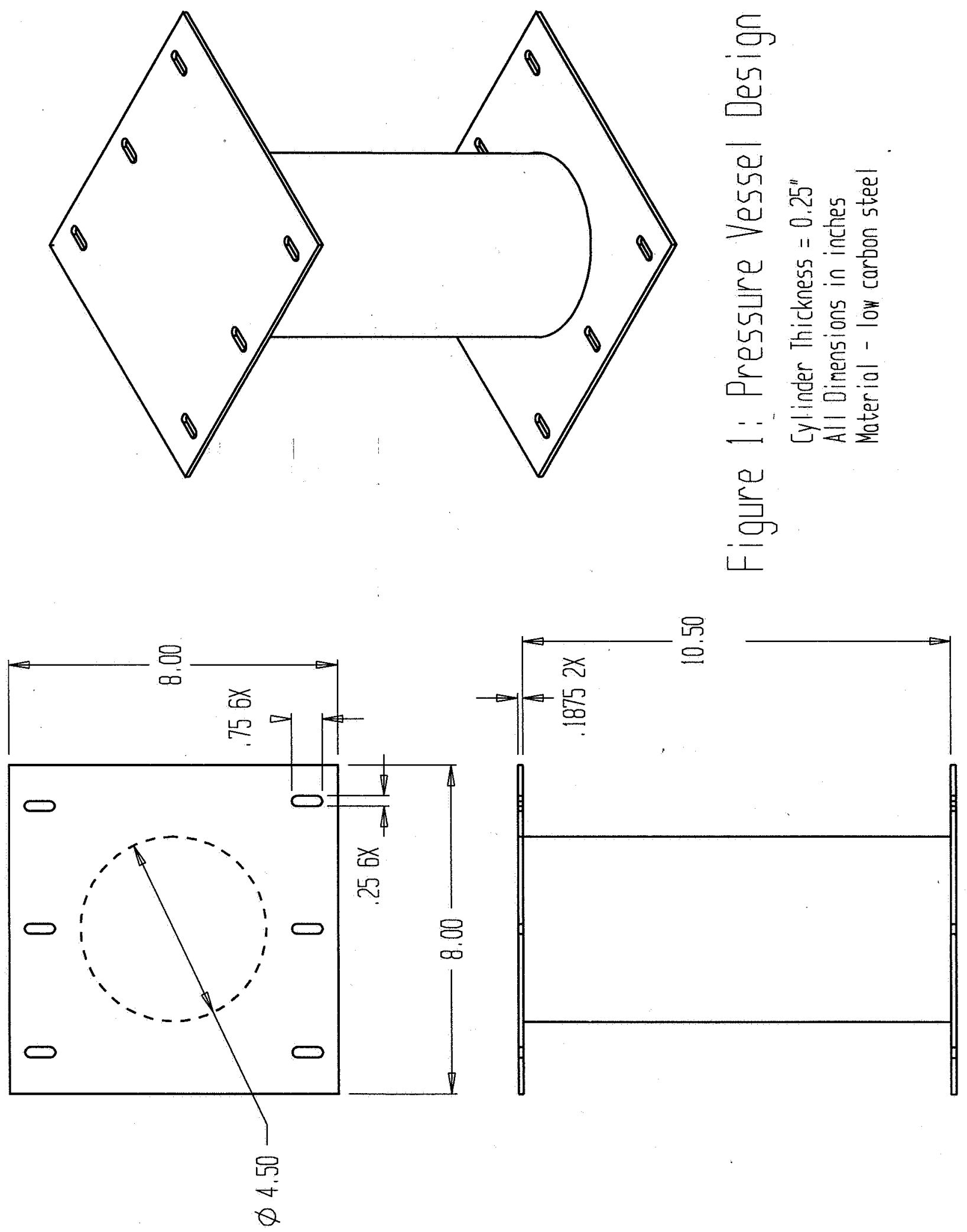